\title{
Prediction of Human Pharmacokinetics Profile of Monoclonal Antibody Using hFcRn Transgenic Mouse Model
}

\author{
Genki Nakamura, ${ }^{a, b}$ Kazuhisa Ozeki, ${ }^{*, a}$ Hiroaki Takesue,${ }^{a}$ Mitsuyasu Tabo, ${ }^{a}$ and \\ Ken-ichi Hosoya ${ }^{b}$ \\ ${ }^{a}$ Research Division, Chugai Pharmaceutical Co., Ltd.; Gotemba, Shizuoka 412-8513, Japan: and ${ }^{b}$ Department of \\ Pharmaceutics, Graduate School of Medicine and Pharmaceutical Sciences, University of Toyama; Toyama \\ 930-0194, Japan. \\ Received September 24, 2020; accepted December 4, 2020
}

\begin{abstract}
Human pharmacokinetics (PK) profiles of monoclonal antibodies (mAbs) are usually predicted using non-human primates (NHP), but this comes with drawbacks in terms of cost and throughput. Therefore, we established a human PK profile prediction method using human neonatal Fc receptor (hFcRn) transgenic mice (TgM). We administered launched $13 \mathrm{mAbs}$ to hFcRn TgM and measured the concentration in plasma using electro-chemiluminescence immunoassay. This was then used to calculate PK parameters and predict human PK profiles. The mAbs showed a bi-phased elimination pattern, and clearance $(C L)(\mathrm{mL} / \mathrm{d} / \mathrm{kg})$ and distribution volume at steady state $\left(V_{\mathrm{dss}}\right)(\mathrm{mL} / \mathrm{kg})$ ranges were 11.0 to 131 and 110 to 285 , respectively. There was a correlation in half-life at elimination phase $\left(t_{1 / 2 \beta}\right)$ between $h F c R n$ TgM and humans for $10 \mathrm{mAbs}$ showing $C L$ of more than $80 \%$ in the elimination phase $\left(R^{2}=0.714\right)$. Human $t_{1 / 2 \beta}$ was predicted using $\mathrm{hFcRn} \mathrm{TgM}$ $t_{1 / 2 \beta} ; 9$ out of $10 \mathrm{mAbs}$ were within 2 -fold the actual values, and all $\mathrm{mAbs}$ were within 3 -fold. Regarding the predicted $C L$ values, 7 out of $10 \mathrm{mAbs}$ were within 2 -fold the human values and all $\mathrm{mAbs}$ were within 3-fold. Furthermore, even on day 7 the predicted $C L$ values of 8 out of $10 \mathrm{mAbs}$ were within 2 -fold the observed value, with all mAbs within 3-fold. These results suggest human PK profiles can be predicted using hFcRn TgM data. These methods can accelerate the development of antibody drugs while also reducing cost and improving throughput.
\end{abstract}

Key words preclinical pharmacokinetics; simulation; monoclonal antibody; clinical pharmacokinetics; human neonatal $\mathrm{Fc}$ receptor ( $\mathrm{hFcRn}$ ) transgenic mouse

\section{INTRODUCTION}

There are numerous biologic drugs on the market today, spanning a wide variety of disease targets from cancers to infections to chronic diseases. ${ }^{1,2}$ As a biologic drug, the monoclonal antibody $(\mathrm{mAb})$ is powerful, combining high specificity, high affinity and a long-half-life. The long half-life is achieved because mAbs can avoid lysosomal degradation by binding to neonatal $\mathrm{Fc}$ receptors $(\mathrm{FcRn})$ under acidic conditions and dissociating at the neutral $\mathrm{pH}$ on the cell surface. ${ }^{3)}$ This long half-life reduces the frequency of administration, giving patients a better QOL.

To realize these mAbs, researchers must first accurately predict human pharmacokinetics (PK) in the non-clinical stage using high-throughput methods. ${ }^{4}$ The ratio at which antigen is neutralized is important for efficacy and safety, not only for predicting exposure, but also for predicting the PK profiles that capture plasma concentration over time. ${ }^{5,6)}$

Many non-clinical systems for predicting human PK prediction utilize non-human primates (NHP),${ }^{7-10)}$ rabbits, ${ }^{11)}$ minipigs ${ }^{12)}$ and $\mathrm{hFcRn}$ transgenic mice $(\mathrm{hFcRn} \mathrm{TgM})^{13-15)}$ for in vivo studies, and the FcRn column for in vitro. ${ }^{16,17)}$ Most of these methods isolate a single parameter, such as clearance $(C L)$ or half-life, and methods that predict overall PK profiles without using NHPs are rare. ${ }^{18)}$ For example, while the Dedrick plot can predict human PK profiles, it requires data from NHPs, and their use can be laborious and expensive. ${ }^{19,20)}$ Tam et al. reported that $7 \mathrm{mAbs}$ showed a robust correlation of $C L$ be- tween human and hFcRn TgM (line 32), indicating that $\mathrm{hFcRn}$ $\mathrm{TgM}$ is suitable as a non-clinical PK evaluation systems. ${ }^{13)}$ While reports show that hFcRn TgM can be successfully used to predict single PK parameters in humans, such as $C L$ and half-life, there have been few attempts to predict full human PK profiles. ${ }^{21)}$ The distribution of $\mathrm{mAb}$ is affected by physiological factors because of high hydrophilicity and large molecular weight. Unlike humans, rodents have faster blood flow and other physiological differences, so it is difficult to predict the human PK profile using a Dedrick plot as with NHPs. ${ }^{22)}$

The mAb PK profile after intravenous (i.v.) administration showed bi-phased pattern for the linear region in humans which could be expressed as two-compartment model. ${ }^{23)}$ Four parameters (K10, K12, K21, V1) are needed to predict human PK profiles. In a previous study, we reported a prediction method which uses the half-life in NHP and the human geometric mean values for $\mathrm{K} 12, \mathrm{~K} 21, \mathrm{~V} 1$ and $V_{\mathrm{dss}}{ }^{24)}$ This method may be able to predict human PK profile simply by using halflife in hFcRn TgM. Substituting hFcRn TgM for NHP would significantly improve high-throughput screening, reduce the required amount of $\mathrm{mAb}$, and lower costs in the non-clinical stages. ${ }^{25,26)}$

In this study, we explore a method for predicting human PK profile using hFcRn TgM (line 32). ${ }^{27}$ ) Thirteen launched antibodies were intravenously co-administered with intravenous immunoglobulin (IVIG) to hFcRn TgM, and blood was collected at $5 \mathrm{~min}, 7 \mathrm{~h}$, and 1, 2, 3, 7, 13 (or 14), 21, and $28 \mathrm{~d}$ after dosing, and the plasma concentrations were measured 
by an electro-chemiluminescence immunoassay (ECLIA) in which the antigen was immobilized on ECLIA plates. Noncompartment analysis (NCA) and two-compartment analysis were performed using the measured plasma concentration, which was then used to obtain the PK parameters. We used these hFcRn TgM parameters to predict the human PK profile; this was accomplished with the plasma concentration from just day 7 after dosing, thus minimizing the pain of animals and shortening the evaluation period.

\section{MATERIALS AND METHODS}

Data Collection The 13 therapeutic mAbs used in this study had all been approved in Japan by the end of 2019. ${ }^{28-37)}$ These target a wide variety of antigens. The PK of mAbs with linear PK in humans were analyzed and their parameter values are listed in Supplementary 1. When two-compartment model parameters could not be obtained from literature, twocompartment model analysis was conducted using the data captured for serum/plasma mean concentration versus time using digitizer software UnGraph Version 5.0 (HULINKS, Tokyo, Japan).

PK Study in hFcRn TgM Pharmacokinetic mAb studies were conducted using male human FcRn homozygous transgenic mice (line 32) (B6. mFcRn -/-. hFcRn Tg line $32+/+$ mouse, Jackson Laboratories, Bar Harbor, ME, U.S.A.). Each $\mathrm{mAb}(10 \mathrm{mg} / \mathrm{kg})$ was administered intravenously to $\mathrm{hFcRn}$ $\operatorname{TgM}(n=3)$ via the tail vein with human intravenous immunoglobulin, IVIG $(1 \mathrm{~g} / \mathrm{kg})$. Blood samples were collected in heparinized tubes from the jugular vein at $5 \mathrm{~min}, 7 \mathrm{~h}, 1,2,3$, 7, 13 (or 14), 21, and $28 \mathrm{~d}$ after administration, and plasma was obtained by centrifugation at $20400 \times \boldsymbol{g}$ for $10 \mathrm{~min}$ at $4{ }^{\circ} \mathrm{C}$. All animal experiments in this study were performed according to the Guidelines for the Care and Use of Laboratory Animals at Chugai Pharmaceutical Co., Ltd.

Bioanalysis of Plasma Samples Plasma concentrations of mAbs were determined by an ECLIA using each antigen (Integrin $\alpha 4 \beta 7$, interleukin (IL) $5 \mathrm{R} \alpha$, IL6R $\alpha$, and BLyS, R\&D Systems, Minneapolis, MN, U.S.A.; IL4R $\alpha$, IL12, IL23 $\alpha$, tumor necrosis factor $\alpha(\mathrm{TNF} \alpha)$, vascular endothelial growth factor receptor 2 (VEGFR2), receptor activator of nuclear factor kappa-B ligand (RANKL), and Sclerostin, BioVision, Milpitas, CA, U.S.A.). Three-hundreds eighty four-well ECLIA plates (MESO SCALE DIAGNOSTICS, Rockville, $\mathrm{MD}$, U.S.A.) were incubated with antigen solution in phosphate buffered saline (PBS) at $5^{\circ} \mathrm{C}$ overnight. Plates were washed with wash buffer (phosphate buffered saline containing $0.05 \%$ Tween 20 , PBST) and blocked with $0.5 \%$ bovine serum albumin (Roche Applied Science, Penzberg, Germany) and 10\% Block Ace (Dainippon Sumitomo Pharma, Osaka, Japan) in Tris-buffered saline containing $0.05 \%$ Tween20, TBS-T (Merck KGaA, Darmstadt, Germany). Standards and plasma samples were diluted in blocking buffer and added to plates. After $1 \mathrm{~h}$ incubation, plates were washed, followed by incubation with biotinylated goat anti-human immunoglobulin G (IgG) (Jackson ImmunoResearch Inc., West Grove, PA, U.S.A.) for $1 \mathrm{~h}$. After washing plates, sTAG-streptavidin was added and incubated for $1 \mathrm{~h}$. Plates were measured on the Sector Imager S600 (MESO SCALE DIAGNOSTICS) after adding Read buffer T. Plasma concentrations of each sample were determined from a standard curve using a 4-parameter nonlinear regression program in Softmax Pro software (Molecular Devices, San Jose, CA, U.S.A.).

PK Data Analysis The plasma mean concentration-time profiles after IV administration were analyzed using the twocompartment open model with Phoenix WinNonlin version 8.0 (Certara Inc., Princeton NJ, U.S.A.). Plasma concentration $(C)$ declined in a bi-exponential fashion using first macroconstant $(A)$, second macro-constant $(B)$, first rate constant $(\alpha)$, second rate constant $(\beta)$.

$$
C=A \times e^{-\alpha t}+B \times e^{-\beta t}
$$

The central elimination rate $(\mathrm{K} 10)$, central to peripheral distribution rate $(\mathrm{K} 12)$, and peripheral to central distribution rate (K21) were obtained using the following equations:

$$
\begin{gathered}
\mathrm{K} 21=(A \times \beta+B \times \alpha) /(A+B) \\
\mathrm{K} 10=(\alpha \times \beta) / \mathrm{K} 21 \\
\mathrm{~K} 12=\alpha+\beta-\mathrm{K} 21-\mathrm{K} 10 \\
\mathrm{~V} 2=(\mathrm{K} 12 \times \mathrm{V} 1) / \mathrm{K} 21
\end{gathered}
$$

The distribution volume in the central compartment (V1) was calculated as $\operatorname{Dose} /(A+B)$; the distribution volume at steady state $\left(V_{\mathrm{dss}}\right)$ was calculated as $\mathrm{V} 1+\mathrm{V} 2$; half-life at elimination phase $\left(t_{1 / 2 \beta}\right)$ was calculated as $\operatorname{Ln}(2) / \beta$. The area under the curve $(A U C)$ was calculated by the following equations:

$$
\begin{gathered}
A U C_{\text {inf }}=A U C_{\alpha}+A U C_{\beta} \\
A U C_{\alpha}=A / \alpha \\
A U C_{\beta}=B / \beta
\end{gathered}
$$

The total body $C L$ was estimated as Dose $/ A U C_{\text {inf. }}$.

Human PK Prediction Using $\boldsymbol{t}_{1 / 2 \boldsymbol{\beta}}$ in hFcRn TgM Using hFcRn TgM $t_{1 / 2 \beta}$, the value of human $t_{1 / 2 \beta}$ was predicted using the linear approximation among 10 mAbs:

$$
t_{1 / 2 \beta \text { human }}=1.89 \times t_{1 / 2 \beta \mathrm{TgM}}+7.18
$$

The values of $\mathrm{V} 1_{\text {human }}, \mathrm{K} 12_{\text {human }}, \mathrm{K} 21_{\text {human }}$ were $45.1 \mathrm{~mL} / \mathrm{kg}$, $0.275,0.355 \mathrm{~d}^{-1}$, respectively. $\mathrm{K} 10_{\text {human }}$ was calculated from the following equation ${ }^{24)}$ :

$$
\mathrm{K} 10_{\text {human }}=1.80 \times \mathrm{LN}(2) / t_{1 / 2 \beta \text { human }}
$$

To simulate the human plasma concentration-time profile after IV, the two-compartment model described in Eq.1 was used.

$$
\begin{aligned}
& \alpha= \mathrm{K} 10+\mathrm{K} 12+\mathrm{K} 21 \\
&+\sqrt{(\mathrm{K} 10+\mathrm{K} 12+\mathrm{K} 21)^{2}-4 \times \mathrm{K} 21 \times \mathrm{K} 10} \\
& \beta= \mathrm{K} 10+\mathrm{K} 12+\mathrm{K} 21 \\
&- \sqrt{(\mathrm{K} 10+\mathrm{K} 12+\mathrm{K} 21)^{2}-4 \times \mathrm{K} 21 \times \mathrm{K} 10} \\
& \mathrm{~A}=\operatorname{Dose} \times(\mathrm{K} 21-\alpha) / \mathrm{V} 1 \times(\beta-\alpha) \\
& \mathrm{B}=\operatorname{Dose} \times(\mathrm{K} 21-\beta) / \mathrm{V} 1 \times(\alpha-\beta)
\end{aligned}
$$

The total body $C L_{\text {human }}$ was estimated as $\mathrm{K} 10_{\text {human }} \times \mathrm{V} 1_{\text {human }}$.

Human PK Prediction Using $C_{\text {day } 7}$ in hFcRn TgM

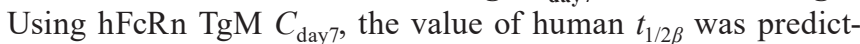
ed using the equation below. Lower limited of quantification 
Table 1. Pharmacokinetic Parameters in hFcRn TgM after Intravenous Administration (13 mAb)

\begin{tabular}{|c|c|c|c|c|c|c|}
\hline Parameter unit & K10 day ${ }^{-1}$ & K12 day ${ }^{-1}$ & $\mathrm{~K}^{21}$ day $^{-1}$ & $\mathrm{~V} 1 \mathrm{~mL} / \mathrm{kg}$ & $C L \mathrm{~mL} / \mathrm{d} / \mathrm{kg}$ & $V_{\mathrm{dss}} \mathrm{mL} / \mathrm{kg}$ \\
\hline Vedolizumab & 2.12 & 1.72 & 1.95 & 58.4 & 124 & 110 \\
\hline Benralizumab & 1.55 & 0.831 & 0.687 & 66.7 & 104 & 147 \\
\hline Romosozumab & 0.825 & 1.64 & 2.68 & 78.6 & 64.8 & 127 \\
\hline Risankizumab & 0.780 & 0.846 & 0.384 & 63.2 & 49.3 & 202 \\
\hline Adalimumab & 0.608 & 2.74 & 1.98 & 76.5 & 46.5 & 183 \\
\hline Ustekinumab & 0.303 & 2.96 & 1.07 & 45.1 & 13.7 & 170 \\
\hline Denosumab & 0.190 & 4.01 & 1.89 & 67.8 & 12.9 & 212 \\
\hline Guselkumab & 1.29 & 2.07 & 1.79 & 101 & 131 & 219 \\
\hline Sarilumab & 0.127 & 2.67 & 1.31 & 86.4 & 11.0 & 263 \\
\hline Ramucirumab & 0.428 & 3.04 & 1.16 & 63.5 & 27.1 & 230 \\
\hline Belimumab & 0.571 & 16.2 & 14.7 & 91.4 & 52.1 & 192 \\
\hline Dupilumab & 0.248 & 3.28 & 1.89 & 88.4 & 21.9 & 242 \\
\hline Golimumab & 0.346 & 1.19 & 0.512 & 85.8 & 29.7 & 285 \\
\hline Geometric mean & 0.529 & 2.35 & 1.51 & 73.2 & 38.8 & 192 \\
\hline CV (\%) & 101 & 90.0 & 111 & 22.5 & 103 & 28.6 \\
\hline
\end{tabular}

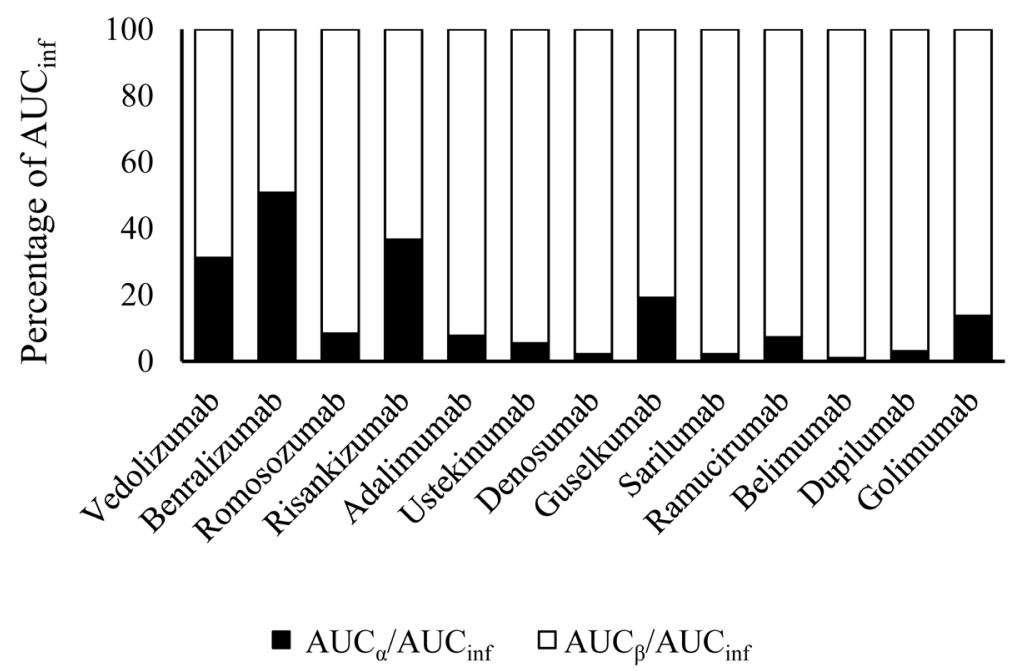

Fig. 1. Two-Compartment Model Analysis of Monoclonal Antibodies after Intravenous Administration in hFcRn TgM

Fraction percentages of exposure in $\mathrm{hFcRn} \operatorname{TgM}$ for the distribution phase $\left(A U C_{\alpha}\right)$ and elimination phase $\left(A U C_{\beta}\right)$, respectively.

(LLOQ) values were used when $C_{\text {day } 7}$ was less than LLOQ.

$$
t_{1 / 2 \beta \text { human }}=0.795 \times C_{\text {day } 7 \mathrm{TgM}}+9.22
$$

Subsequent calculations were performed as described above (Eq. 11 to 14).

\section{RESULTS}

mAb PK in hFcRn TgM PK parameters in hFcRn TgM from NCA and two compartment model analysis are shown in Table 1. Geometric mean and CV (\%) of K10 (day $\left.{ }^{-1}\right), \mathrm{K} 12$ $\left(\right.$ day $\left.^{-1}\right), \mathrm{K} 21\left(\right.$ day $\left.^{-1}\right)$, and $\mathrm{V} 1(\mathrm{~mL} / \mathrm{kg})$, which are microscopic parameters of the two compartment model, were 0.529, 2.35, 1.51, 73.2 and 101, 90.0, 111, 22.5, respectively. For the NCA parameters, $C L(\mathrm{~mL} / \mathrm{d} / \mathrm{kg})$ and $V_{\mathrm{dss}}(\mathrm{mL} / \mathrm{kg})$, the geometric mean, and CV (\%) were 38.8 and 192, 103, 28.6, respectively. There was little difference in distribution volume among the 13 mAbs. The ratios of $A U C_{\beta} / A U C_{\text {inf }}(\%)$ in vedolizumab, benralizumab, and risankizumab were 68.8, 49.2, and 63.3, and the others were over $80 \%$ (Fig. 1).
Correlation between hFcRn TgM $t_{1 / 2 \beta}$ and Human $t_{1 / 2 \beta}$ We examined the correlation in $t_{1 / 2 \beta}$ between $\mathrm{hFcRn} \mathrm{TgM}$ and human. For the $13 \mathrm{mAbs}$, linear approximation was calculated with following equation: human $t_{1 / 2 \beta}=1.39 \times \mathrm{hFcRn} \mathrm{TgM}$ $t_{1 / 2 \beta}+13.2$ and $R^{2}$ was 0.409 (Fig. 2A). Next, linear approximation was performed excluding vedolizumab, benralizumab, and risankizumab, which all showed $A U C_{\beta} / A U C_{\text {inf }}$ of less than $80 \%$. In the equation, human $t_{1 / 2 \beta}=1.89 \times \mathrm{hFcRn} \mathrm{TgM}$ $t_{1 / 2 \beta}+7.18$ and $R^{2}$ value was 0.714 (Fig. 2B).

Prediction of Human PK Profile Using $t_{1 / 2 \beta}$ in hFcRn TgM Human $t_{1 / 2 \beta}$ and $C L$ values were predicted using the equation of linear approximation between hFcRn TgM $t_{1 / 2}$ and human $t_{1 / 2 \beta}$ for $10 \mathrm{mAbs}$. For human $t_{1 / 2 \beta}$ values, 9 out of $10 \mathrm{mAbs}$ were predicted within 2-fold the actual value. With 1 $\mathrm{mAb}$ showing a 2.23 fold higher value, all $10 \mathrm{mAbs}$ were well within 3 -fold (Fig. 3A). We plugged the predicted human $t_{1 / 2 \beta}$ values into the half-life method to generate the human PK profile (Supplementary 2). There was a less than 2-fold difference between the predicted and observed $C L$ of $7 \mathrm{mAbs}$, and a less than 3-fold difference for all mAbs (Fig. 3B).

Correlation between hFcRn TgM $C_{\text {day } 7}$ to Human $t_{1 / 2 \beta}$ 
A

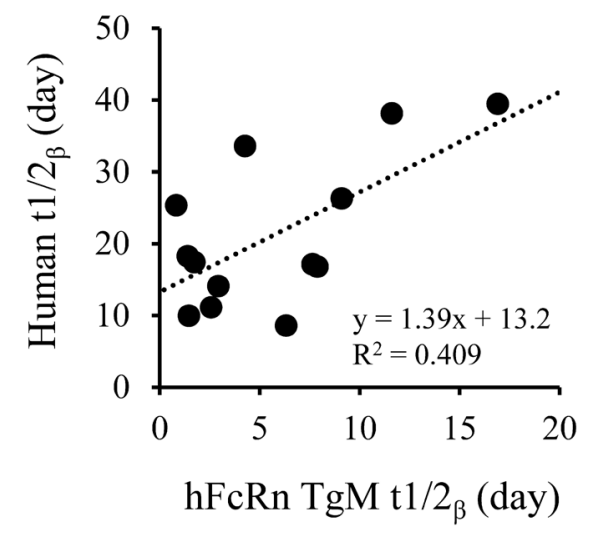

B

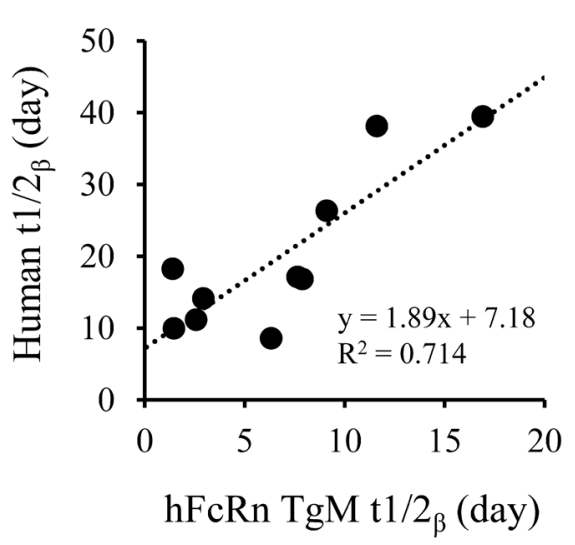

Fig. 2. Correlation in $t_{1 / 2 \beta}$ between hFcRn TgM and Human

(A) Comparison of half-life during elimination phase $\left(t_{1 / 2 \beta}\right)$ between $\mathrm{hFcRn} \operatorname{TgM}$ and humans for $13 \mathrm{mAbs}$. The dotted line is the regression (linear regression) line. (B) Comparison of half-life during elimination phase $\left(t_{1 / 2 \beta}\right)$ between $\mathrm{hFcRn} \mathrm{TgM}$ and humans for $10 \mathrm{mAbs}$. The dotted line is the regression (linear regression) line.

The correlation between $C_{\text {day } 7}$ in hFcRn TgM and human $t_{1 / 2 \beta}$ was investigated. For $13 \mathrm{mAbs}$, linear approximation was calculated by the following equation: human $t_{1 / 2 \beta}=0.517 \times \mathrm{hFcRn}$ $\operatorname{TgM} C_{\text {day } 7}+15.4$ and $R^{2}$ value was 0.199 (Fig. 4A). Next, linear approximation was performed excluding vedolizumab, romosozumab, and risankizumab, which showed around less than $80 \% \quad A U C_{\beta} / A U C_{\text {inf. }}$. The equation was human $t_{1 / 2 \beta}=0.795 \times \mathrm{hFcRn} \operatorname{TgM} C_{\text {day } 7}+9.22$ and $R^{2}$ value was 0.425 (Fig. 4B).

Prediction of Human PK Profile Using $C_{\text {day } 7}$ in hFcRn TgM Human $t_{1 / 2 \beta}$ and $C L$ values were predicted using the equation of linear approximation between $\mathrm{hFcRn} \operatorname{TgM} C_{\text {day } 7}$ and human $t_{1 / 2 \beta}$ for $10 \mathrm{mAbs}$. For human $t_{1 / 2 \beta}$ values, 9 out of $10 \mathrm{mAbs}$ were predicted within 2-fold the actual value. With $1 \mathrm{mAb}$ showing a 2.46 higher value, all $10 \mathrm{mAbs}$ were within 3 -fold (Fig. 5A). We plugged the predicted human $t_{1 / 2 \beta}$ values into the half-life method to generate the human PK profile (Supplementary 3). There was a less than 2-fold difference between the predicted and observed $C L$ of $8 \mathrm{mAbs}$, and a less than 3-fold difference for all mAbs (Fig. 5B).

\section{DISCUSSION}

Human PK prediction is essential in the development of
A

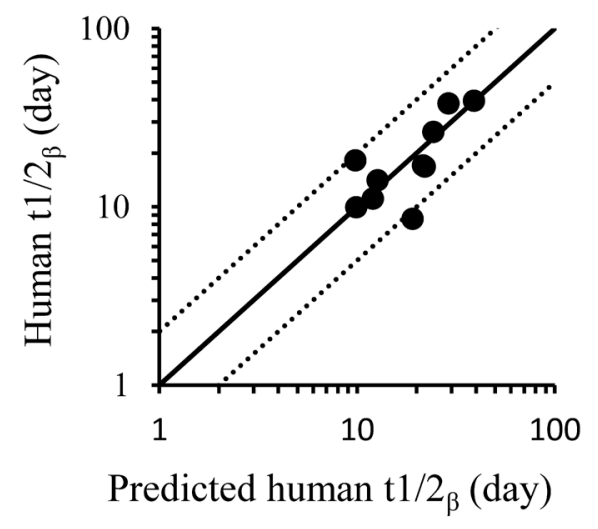

$\mathrm{B}$

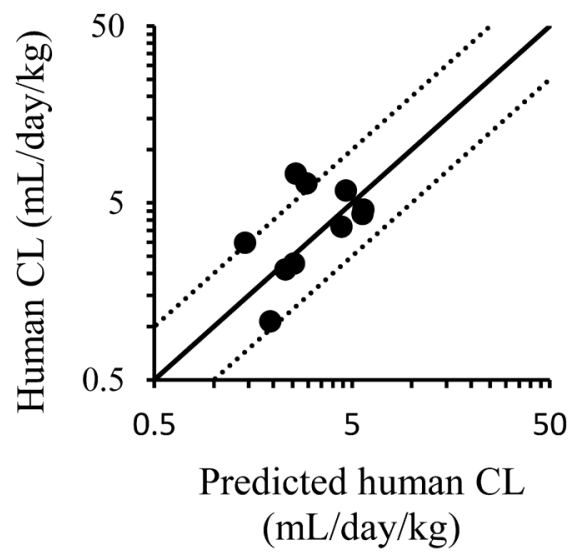

Fig. 3. Comparison of Human PK Predicted Using $t_{1 / 2 \beta}$ in hFcRn TgM and Observed Human PK

(A) Predicted values for human half-life during elimination phase $\left(t_{1 / 2 \beta}\right)$ were plotted against observed values for human $t_{1 / 2 \beta}$. Human $t_{1 / 2 \beta}$ was predicted from $\mathrm{hFcRn} \mathrm{TgM}$ data using linear regression. Solid diagonal line represents the line of unity. The dotted lines represent two-fold range above and below the line of unity. (B) Predicted values for human $C L$ were plotted against observed values. Human $C L$ was predicted from hFcRn TgM data. Solid diagonal line represents the line of unity. The dotted lines represent two-fold range above and below the line of unity.

$\mathrm{mAb}$ drugs, and it requires accurate high throughput evaluation and a deep consideration for the animals involved in these experiments. Determining the human PK profile is critical for drug efficacy and safety because it allows us to calculate the time-dependent receptor occupancy ratio. ${ }^{6)}$ Human PK profiles are usually predicted using NHP data, but this is expensive, laborious, and difficult for the animals. ${ }^{18)}$ There is a great need for a more efficient - but still accurate — substitute which utilizes rodents or in vitro systems. ${ }^{25,26)}$ To address this, we attempted to predict human PK profiles using hFcRn TgM data and the half-life method. ${ }^{24)}$

In this study, we analyzed PK data on 13 mAbs, revealing a bi-phased elimination pattern in hFcRn TgM. K12 and K21 values ranged widely, showing an approximately $1: 1$ correlation. We think this is largely because the distribution volume of the central and peripheral were almost the same among mAbs. $C L$ values showed a good inverse correlation with $t_{1 / 2 \beta}$, consistent with the large effect of the terminal phase on clearance. The disappearance of antibodies in humans 
A

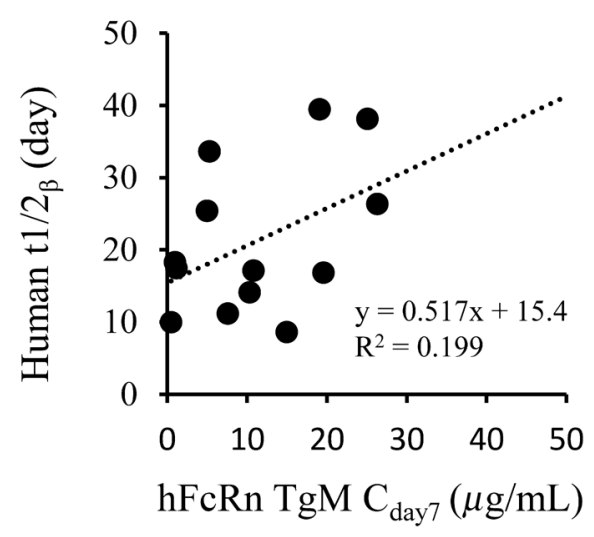

B

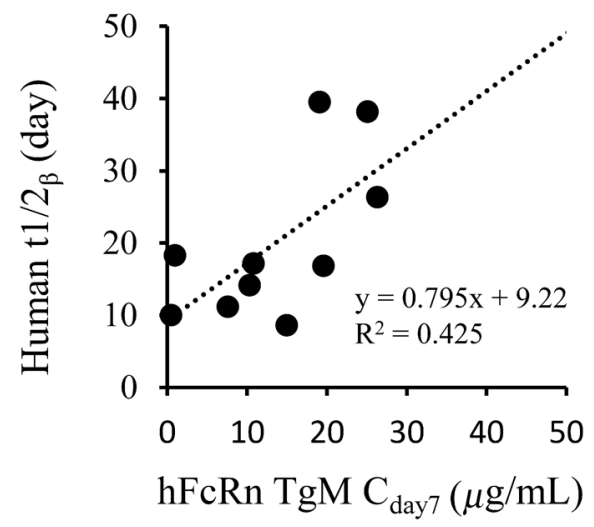

Fig. 4. Correlation between $C_{\text {day }}$ in hFcRn $\operatorname{TgM}$ and $t_{1 / 2 \beta}$ in Humans

(A) Comparison of $C_{\text {day } 7}$ in hFcRn $\operatorname{TgM}$ and half-life at elimination phase $\left(t_{1 / 2 \beta}\right)$ in humans for $13 \mathrm{mAbs}$. The dotted line is the regression (linear regression) line. (B) Comparison of $C_{\text {day } 7}$ in hFcRn TgM and half-life at elimination phase $\left(t_{1 / 2 \beta}\right)$ in humans for $10 \mathrm{mAbs}$. The dotted line is the regression (linear regression) line.

is divided into a receptor-mediated nonlinear region and a nonspecific linear region, and the linear region is reported to exhibit biphasic kinetics. ${ }^{23,24)}$ In human $\mathrm{PK}, C L$ and $t_{1 / 2 \beta}$ are inversely correlated and exposure during the elimination phase contributes most to $C L$. The difference in distribution volume (V1 and $V_{\text {dss }}$ ) among mAbs is small, and K12 and K21 are proportional. This is almost the same in both humans and hFcRn TgM. We think this is because of the high molecular weight, high hydrophilicity, and the mechanistic features of FcRn recycling. ${ }^{27)}$

Ten out of the $13 \mathrm{mAbs}$ used in this study showed a good correlation in $t_{1 / 2 \beta}$ between $\mathrm{hFcRn} \mathrm{TgM}$ and humans, with vedolizumab, benralizumab and risankizumab as the exceptions. The human $t_{1 / 2 \beta}$ values predicted based on this correlation were within 2-fold of the actual values for $90 \%$ of the estimates. This suggests $\mathrm{hFcRn} \operatorname{TgM}$ data is sufficient for predicting human PK profiles. On the other hand, the correlation in $t_{1 / 2 \beta}$ between $\mathrm{hFcRn} \operatorname{TgM}$ and humans was poor when data for all 13 mAbs was included. The hFcRn TgM model can be used to non-clinically evaluate half-life via the hFcRn recycling system. The majority of $A U C_{\text {inf }}$ was $A U C_{\beta}$, meaning that $C L$ depends on $t_{1 / 2 \beta}$, and that $t_{1 / 2 \beta}$ therefore reflects FcRn recycling. Excluding mAbs with an $A U C_{\beta}$ contribution of $70 \%$ or less (vedolizumab, romosozumab, and risankizumab),
A

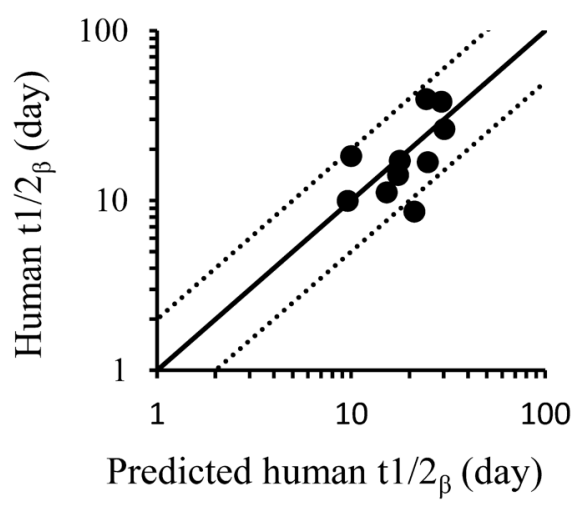

$\mathrm{B}$

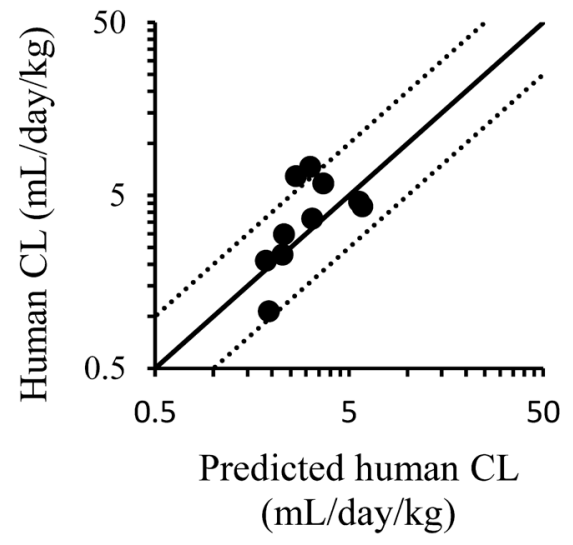

Fig. 5. Comparison of Predicted Human PK Using $C_{\text {day }}$ in hFcRn TgM and Observed Human PK

(A) Predicted values for human half-life at elimination phase $\left(t_{1 / 2 \beta}\right)$ were plotted against observed values for human $t_{1 / 2 \beta}$. Human $t_{1 / 2 \beta}$ was predicted from $\mathrm{hFcRn}$ $\operatorname{TgM} C_{\text {day }}$ data using linear regression. Solid diagonal line represents the line of unity. The dotted lines represent two-fold range above and below the line of unity. (B) Predicted values for human $C L$ were plotted against observed values for human $C L$. Human $C L$ was predicted from $\mathrm{hFcRn} \mathrm{TgM}$ data. Solid diagonal line represents the line of unity. The dotted lines represent two-fold range above and below the line of unity.

improved the correlation of $t_{1 / 2 \beta}$ between hFcRn TgM and human. Therefore, for a small proportion of $A U C_{\beta}$ in $A U C_{\text {inf }}$, factors other than FcRn recycling contribute to $t_{1 / 2 \beta}$ in $\mathrm{hFcRn}$ TgM. Although human $t_{1 / 2 \beta}$ could be predicted from hFcRn $\mathrm{TgM}$, it might be necessary to confirm the contribution of $A U C_{\beta}$ and apply this correlation.

Non-clinical studies using NHPs are expensive, requiring costly antibody resources. Rodent studies would be more efficient, but PK results using wild type mice cannot be appropriately extrapolated to humans because of the different affinities against mouse and human FcRn. ${ }^{38)}$ In contrast, hFcRn TgM, in which the mFcRn is replaced with human FcRn, correlates well with human PK parameters. ${ }^{13,27)}$ Although human PK has been predicted for single parameters, the prediction of full PK profiles are rarely reported. ${ }^{21)}$ One reason for this is that hFcRn TgM and humans have a lot of physiological differences, such as with blood flow. ${ }^{22)}$ This makes it difficult to adapt the Dedrick plot, commonly used with NHPs to predict 
human PK, to rodents. ${ }^{19,20)}$ Our new method has been reported to predict human PK profile using only the half-life of monkey. ${ }^{24)}$ Because the $t_{1 / 2}$ of hFcRn TgM correlates with the $t_{1 / 2}$ of humans, this method should also apply here. Previous studies support the successful prediction of human PK profiles using $t_{1 / 2 \beta}$ in human FcRn TgM. ${ }^{13)}$

To improve animal welfare and shorten the evaluation period, we predicted human PK profile using only the plasma concentration in hFcRn TgM on day 7. With just one time point needed, blood is sampled less often, which advances the refinement aim of the $3 \mathrm{Rs}$ (replacement, reduction, and refinement of animal use in research). ${ }^{25,26)}$ The correlation between plasma concentration on day 7 and human $t_{1 / 2 \beta}$ itself was not ideal, and $R^{2}$ value was not improved by logarithmic approximation. (data not shown) This might be because the LLOQ value was adapted to $C_{\text {day } 7}$ in the case of LLOQ at day 7, and a continued effort to achieve more sensitive detection methods is required. The predicted human $t_{1 / 2 \beta}$ values using this correlation were within 2-fold of the actual values for $90 \%$ of estimates. In addition, using only the plasma concentration on day 7, the predicted $C L$ for $80 \%$ of the evaluated mAbs was within 2 -fold the actual human values. Further studies are needed to fully ensure the accuracy of this method.

In evaluating the PK of $\mathrm{hFcRn} \mathrm{TgM}$ in this study, IVIG $(1 \mathrm{~g} / \mathrm{kg})$ was co-administered to achieve a concentration of about a $20 \mathrm{mg} / \mathrm{mL}$ in plasma to reflect the human situation. Albumin was co-administered in other reports, and improving the hFcRn TgM study conditions might raise prediction accuracy. ${ }^{13)}$ However, animals are still involved in this approach and it might be possible to progress to a purely in vitro system. Also, in vitro systems are currently only used for ranking and have rarely been adapted to predict human PK profiles, so more advanced in vitro systems are needed. ${ }^{16,17,39)}$ Regarding FcRn, NHPs and humans have a close homology with humans, but hFcRn TgM have exactly the same, so it might be possible to more accurately predict human PK profiles using the mice. $^{27)}$

In this study, we demonstrated that human PK profiles can be predicted using hFcRn TgM. This could potentially make the non-clinical development of drugs more efficient.

Acknowledgment We thank Jacob Davis at Chugai Pharmaceutical Co., Ltd. for advice in the preparation and language editing of this manuscript.

Conflict of Interest The authors declare no conflict of interest.

Supplementary Materials The online version of this article contains supplementary materials.

\section{REFERENCES}

1) Ecker DM, Jones SD, Levine HL. The therapeutic monoclonal antibody market. $m A b s, 7,9-14$ (2015).

2) Kaplon H, Muralidharan M, Schneider Z, Reichert JM. Antibodies to watch in 2020. $m A b s, \mathbf{1 2}, 1703531$ (2020).

3) Tabrizi MA, Tseng CM, Roskos LK. Elimination mechanisms of therapeutic monoclonal antibodies. Drug Discov. Today, 11, 81-88 (2006).

4) Haraya K, Tachibana T. Simple approach to accurately predict phar- macokinetics of therapeutic monoclonal antibodies after subcutaneous injection in humans. Clin. Pharmacokinet., 60, 111-120 (2020).

5) Wang J, Iyer S, Fielder PJ, Davis JD, Deng R. Projecting human pharmacokinetics of monoclonal antibodies from nonclinical data: comparative evaluation of prediction approaches in early drug development. Biopharm. Drug Dispos., 37, 51-65 (2016).

6) Gram H. Preclinical characterization and clinical development of ILARIS((R)) (canakinumab) for the treatment of autoinflammatory diseases. Curr. Opin. Chem. Biol., 32, 1-9 (2016).

7) Wang W, Prueksaritanont T. Prediction of human clearance of therapeutic proteins: simple allometric scaling method revisited. Biopharm. Drug Dispos., 31, 253-263 (2010).

8) Deng R, Iyer S, Theil FP, Mortensen DL, Fielder PJ, Prabhu S. Projecting human pharmacokinetics of therapeutic antibodies from nonclinical data: what have we learned? $m A b s, 3,61-66$ (2011).

9) Dong JQ, Salinger DH, Endres CJ, Gibbs JP, Hsu CP, Stouch BJ, Hurh E, Gibbs MA. Quantitative prediction of human pharmacokinetics for monoclonal antibodies: retrospective analysis of monkey as a single species for first-in-human prediction. Clin. Pharmacokinet., 50, 131-142 (2011).

10) Oitate M, Masubuchi N, Ito T, Yabe Y, Karibe T, Aoki T, Murayama N, Kurihara A, Okudaira N, Izumi T. Prediction of human pharmacokinetics of therapeutic monoclonal antibodies from simple allometry of monkey data. Drug Metab. Pharmacokinet., 26, 423430 (2011).

11) Mahmood I. Interspecies scaling of protein drugs: prediction of clearance from animals to humans. J. Pharm. Sci., 93, 177-185 (2004).

12) Zheng Y, Tesar DB, Benincosa L, et al. Minipig as a potential translatable model for monoclonal antibody pharmacokinetics after intravenous and subcutaneous administration. $m A b s, 4,243-255$ (2012).

13) Tam SH, McCarthy SG, Brosnan K, Goldberg KM, Scallon BJ. Correlations between pharmacokinetics of IgG antibodies in primates vs. FcRn-transgenic mice reveal a rodent model with predictive capabilities. $m A b s, \mathbf{5}, 397-405$ (2013).

14) Haraya K, Tachibana T, Nanami M, Ishigai M. Application of human FcRn transgenic mice as a pharmacokinetic screening tool of monoclonal antibody. Xenobiotica, 44, 1127-1134 (2014).

15) Avery LB, Wang M, Kavosi MS, Joyce A, Kurz JC, Fan YY, Dowty ME, Zhang M, Zhang Y, Cheng A, Hua F, Jones HM, Neubert H, Polzer RJ, O'Hara DM. Utility of a human FcRn transgenic mouse model in drug discovery for early assessment and prediction of human pharmacokinetics of monoclonal antibodies. $m A b s, \mathbf{8}, 1064$ 1078 (2016)

16) Schlothauer $T$, Rueger $\mathrm{P}$, Stracke JO, Hertenberger H, Fingas $\mathrm{F}$, Kling L, Emrich T, Drabner G, Seeber S, Auer J, Koch S, Papadimitriou A. Analytical FcRn affinity chromatography for functional characterization of monoclonal antibodies. $m A b s$, 5, 576-586 (2013).

17) Schoch A, Kettenberger H, Mundigl O, Winter G, Engert J, Heinrich J, Emrich T. Charge-mediated influence of the antibody variable domain on FcRn-dependent pharmacokinetics. Proc. Natl. Acad. Sci. U.S.A., 112, 5997-6002 (2015).

18) Iwasaki K, Uno Y, Utoh M, Yamazaki H. Importance of cynomolgus monkeys in development of monoclonal antibody drugs. Drug Metab. Pharmacokinet., 34, 55-63 (2019).

19) Oitate M, Nakayama S, Ito T, Kurihara A, Okudaira N, Izumi T. Prediction of human plasma concentration-time profiles of monoclonal antibodies from monkey data by a species-invariant time method. Drug Metab. Pharmacokinet., 27, 354-359 (2012).

20) Xiang H, Bender BC, Reyes AE 2nd, Merchant M, Jumbe NL, Romero M, Davancaze T, Nijem I, Mai E, Young J, Peterson A, Damico-Beyer LA. Onartuzumab (MetMAb): using nonclinical pharmacokinetic and concentration-effect data to support clinical development. Clin. Cancer Res., 19, 5068-5078 (2013). 21) Betts A, Keunecke A, van Steeg TJ, van der Graaf PH, Avery LB, 
Jones H, Berkhout J. Linear pharmacokinetic parameters for monoclonal antibodies are similar within a species and across different pharmacological targets: a comparison between human, cynomolgus monkey and $\mathrm{hFcRn} \mathrm{Tg} 32$ transgenic mouse using a populationmodeling approach. $m A b s, \mathbf{1 0}, 751-764$ (2018).

22) Davies B, Morris T. Physiological parameters in laboratory animals and humans. Pharm. Res., 10, 1093-1095 (1993).

23) Dirks NL, Meibohm B. Population pharmacokinetics of therapeutic monoclonal antibodies. Clin. Pharmacokinet., 49, 633-659 (2010).

24) Nakamura G, Ozeki K, Nagayasu M, Nambu T, Nemoto T, Hosoya KI. Predicting method for the human plasma concentration-time profile of a monoclonal antibody from the half-life of non-human primates. Biol. Pharm. Bull., 43, 823-830 (2020).

25) Diehl KH, Hull R, Morton D, Pfister R, Rabemampianina Y, Smith D, Vidal JM, van de Vorstenbosch C. A good practice guide to the administration of substances and removal of blood, including routes and volumes. J. Appl. Toxicol., 21, 15-23 (2001).

26) Raje AA, Mahajan V, Pathade VV, Joshi K, Gavali A, Gaur A, Kandikere V. Capillary microsampling in mice: effective way to move from sparse sampling to serial sampling in pharmacokinetics profiling. Xenobiotica, 50, 663-669 (2020).

27) Proetzel G, Roopenian DC. Humanized FcRn mouse models for evaluating pharmacokinetics of human IgG antibodies. Methods, $\mathbf{6 5}$, 148-153 (2014)

28) Vedolizumab PMDA. 2018. 〈https://www.pmda.go.jp/drugs/2018/ P20180713001/index.html, accessed 12 August, 2020.

29) Benralizumab PMDA. 2018. 〈https://www.pmda.go.jp/drugs/2018/ P20180216003/index.html, accessed 12 August, 2020.
30) Romosozumab PMDA. 2019. 〈https://www.pmda.go.jp/drugs/2019/ P20190122003/index.html, accessed 12 August, 2020.

31) Risankizumab PMDA. 2019. 〈https://www.pmda.go.jp/drugs/2019/ P20190329001/index.html, accessed 12 August, 2020.

32) Ustekinumab PMDA. 2011. 〈https://www.pmda.go.jp/drugs/2011/ P201100021/index.html, accessed 12 August, 2020.

33) Denosumab PMDA. 2013. 〈https://www.pmda.go.jp/drugs/2013/ P201300022/index.html, accessed 12 August, 2020.

34) Zhuang Y, Calderon C, Marciniak SJ Jr, Bouman-Thio E, Szapary P, Yang TY, Schantz A, Davis HM, Zhou H, Xu Z. First-in-human study to assess guselkumab (anti-IL-23 mAb) pharmacokinetics/ safety in healthy subjects and patients with moderate-to-severe psoriasis. Eur. J. Clin. Pharmacol., 72, 1303-1310 (2016).

35) Xu C, Su Y, Paccaly A, Kanamaluru V. Population pharmacokinetics of sarilumab in patients with rheumatoid arthritis. Clin. Pharmacokinet., 58, 1455-1467 (2019).

36) Dupilumab PMDA. 2018. 〈https://www.pmda.go.jp/drugs/2018/ P20180129001/index.html, accessed 12 August, 2020.

37) Golimumab PMDA. 2011. 〈https://www.pmda.go.jp/drugs/2011/ P201100114/index.html, accessed 12 August, 2020.

38) Ober RJ, Radu CG, Ghetie V, Ward ES. Differences in promiscuity for antibody-FcRn interactions across species: implications for therapeutic antibodies. Int. Immunol., 13, 1551-1559 (2001).

39) Jones HM, Zhang Z, Jasper P, Luo H, Avery LB, King LE, Neubert H, Barton HA, Betts AM, Webster R. A physiologically-based pharmacokinetic model for the prediction of monoclonal antibody pharmacokinetics from in vitro data. CPT Pharmacometrics Syst. Pharmacol., 8, 738-747 (2019). 